\title{
INDICADORES PRELIMINARES DE MADUREZ FISIOLÓGICA Y COMPORTAMIENTO POSTCOSECHA DEL FRUTO DE AGUACATE MÉNDEZ
}

\section{PRELIMINARY SIGNS OF PHYSIOLOGICAL MATURITY AND POSTHARVEST PERFORMANCE OF MÉNDEZ AVOCADO FRUIT}

\author{
Juan A. Herrera-González', Samuel Salazar-García*, Héctor \\ E. Martínez-Flores ${ }^{3}$ y Jessica E. Ruiz-García ${ }^{3}$
}

\begin{abstract}
'Campo Experimental Uruapan, Instituto Nacional de Investigaciones Forestales, Agrícolas y Pecuarias (INIFAP). Av. Latinoamericana 1101. 60150 Uruapan, Michoacán, México. ${ }^{2}$ Campo Experimental Santiago Ixcuintla, INIFAP. Apdo. Postal 100. 63300, Santiago Ixcuintla, Nayarit, México. Tel. (55) 3871-8700 ext. 84426. ${ }^{3}$ Facultad de Químico Farmacobiología, Universidad Michoacana de San Nicolás de Hidalgo. Tzintzutzan 173. 58240, Col. Matamoros, Morelia, Michoacán, México.
\end{abstract}

*Autor para correspondencia (salazar.samuel@inifap.gob.mx)

\section{RESUMEN}

El aguacate (Persea americana) Méndez tiene gran importancia económica en México, porque gran parte de su cosecha anual se realiza entre junio y septiembre, periodo de poca disponibilidad de fruto de Hass. Aunque la producción y exportación de Méndez se ha incrementado en los últimos años, se desconocen los requisitos mínimos de calidad, como los existentes en Hass. El objetivo de esta investigación fue comparar algunos parámetros de calidad, a la cosecha y en postcosecha, de frutos producidos por la floración de veranootoño de los aguacates Hass y Méndez en Michoacán. La madurez fisiológica se determinó mediante el comportamiento postcosecha de frutos cosechados en muestreos quincenales de mayo a agosto. En madurez fisiológica se cuantificó la respiración y producción de etileno en frutos refrigerados $\left(5.5 \pm 2{ }^{\circ} \mathrm{C}\right.$ y $\left.90 \pm 5 \% \mathrm{HR}\right)$ por $0,7,14$ y $28 \mathrm{~d}$. La madurez fisiológica fue diferente $(P \leq 0.05)$ entre los dos cultivares y se alcanzó 10 meses después de floración, Hass mostró 21.1 \% de materia seca en la pulpa (MS) y Méndez con $22.7 \%$. Aunque la longitud de los frutos de ambos cultivares fue similar, el diámetro ecuatorial fue mayor en Méndez $(6.72 \mathrm{~cm})$ que en Hass $(6.42 \mathrm{~cm})$. El comportamiento postcosecha de Méndez en términos de cambio de color de la piel, producción de etileno, velocidad de respiración, días a madurez de consumo y pérdida de peso del fruto; así como en color, olor, sabor y textura de la pulpa fue similar al de Hass cuando los frutos de ambos cultivares fueron cosechados en madurez fisiológica.

Palabras clave: Persea americana, calidad de fruto, etileno, evaluación sensorial, madurez de cosecha.

\section{SUMMARY}

Méndez avocado has great economic importance in Mexico, because a significant proportion of its annual harvest coincides with limited availability of Hass avocado, from June to September. Although production and export of Méndez avocado has increased in recent years, its minimum quality requirements are not established, unlike Hass avocado. This research compared quality parameters at harvest and post-harvest of Méndez and Hass avocados produced by the Summer-Fall bloom in Michoacán. Physiological maturity was determined by evaluating the postharvest performance of fruit harvested in biweekly sampling from May to August. At physiological maturity, respiration rate and ethylene production were quantified in refrigerated fruit $\left(5.5 \pm 2{ }^{\circ} \mathrm{C}\right.$ and $\left.90 \pm 5 \% \mathrm{RH}\right)$ for $0,7,14$ and $28 \mathrm{~d}$. Physiological maturity was different $(P \leq 0.05)$ between both cultivars, and it was reached 10 months after blooming. Hass avocados contained $21.1 \%$ of pulp dry matter, while
Méndez avocado had $22.7 \%$. Although average fruit length for both cultivars was similar, average equatorial diameter was greater in Mendez avocado $(6.72 \mathrm{~cm})$ than in Hass avocado $(6.42 \mathrm{~cm})$. Post-harvest performance of Mendez avocado, as measured by skin color change, ethylene production, respiration rate, days to maturity of consumption, weight loss of fruit, and pulp characteristics (color, odor, flavor and texture), was similar to Hass avocado performance when the fruit was harvested at physiological maturity..

Index words: Persea americana, fruit quality, ethylene, sensory evaluation, harvest maturity

\section{INTRODUCCIÓN}

El aguacate (Persea americana) Hass es el cultivar que más se produce y consume en el mundo (Ernst, 2007). En Michoacán, debido a la amplia adaptación de este cultivar a diversas altitudes y tipos de clima es posible obtener cosechas todo el año (Salazar-García et al., 2005); sin embargo, de junio a septiembre existe menor producción de fruto, aunque la demanda se mantiene y los precios aumentan (IIlsley-Granich et al., 2011). En este periodo de producción baja de Hass, el aguacate Méndez es una alternativa económica para la industria del aguacate en México.

En climas semicálidos la fenología de Méndez es distinta a la de Hass porque presenta una floración temprana atípica en el verano-otoño. Los frutos de esta floración suelen ser cosechados en los meses de menor producción de Hass (junio-septiembre) (Illsley-Granich et al., 2011). En estos meses de cosecha, los frutos de Hass y Méndez son empacados y almacenados indistintamente para exportación en los mismos contenedores y bajo las mismas condiciones.

Diversas investigaciones en Hass han permitido proponer parámetros de calidad y almacenamiento para su cosecha y comercialización. Se menciona que Hass alcanza

Recibido: 15 de abril de 2016

Aceptado: 23 de noviembre de 2016 
la madurez de cosecha con un mínimo de $21.5 \%$ de materia seca (MS) en la pulpa (mesocarpio) y su temperatura óptima de refrigeración es $5.5^{\circ} \mathrm{C}$ no más de cuatro semanas, con una humedad relativa de 90 a $95 \%$. La tasa de respiración durante la refrigeración es de 10 a $100 \mathrm{~mL} \mathrm{CO}$ $\mathrm{kg}^{-1} \mathrm{~h}^{-1}$ y una producción de etileno a $20^{\circ} \mathrm{C}>100 \mu \mathrm{L}_{2} \mathrm{H}_{4}$ $\mathrm{kg}^{-1} \mathrm{~h}^{-1}$ (Dixon et al., 2005; Huysamer y Maré, 2003; Yearsley et al., 2002).

El aguacate Hass es un fruto climatérico que no produce etileno mientras está adherido al árbol por lo que no madura en esta condición (Hershkovitz et al., 2009a y 2009b); sin embargo, presenta un fuerte aumento en la producción de etileno después de la cosecha y durante la maduración. Las fisiopatías, daños físicos, enfermedades postcosecha, días a madurez de consumo, cambio de color de la piel, pérdida fisiológica de peso y diversos parámetros sensoriales han sido estudiados bajo diferentes condiciones de almacenamiento (Dixon et al., 2008).

No obstante, la importancia del aguacate Méndez aún no es tan fuerte y no cuenta con indicadores de calidad como el Hass. El objetivo de esta investigación fue generar información básica de calidad a la cosecha y en postcosecha en aguacate Méndez, utilizando Hass como referencia.

\section{MATERIALES Y MÉTODOS}

\section{Estudio 1. Madurez de cosecha}

Área de estudio. Se tomaron muestras de frutos provenientes de cuatro huertos de aguacate Méndez y como testigo se incluyó fruto colectado en un huerto de Hass, todos injertados sobre portainjertos de raza mexicana originados por semilla, ubicados en los municipios de Uruapan y Peribán de Ramos, Michoacán (Cuadro 1).

Indicadores de madurez. Se realizaron siete muestreos de fruto, procedentes de la floración de verano-otoño (del 23 al 29 de septiembre de 2011), a intervalos de $15 \mathrm{~d}$. De seis árboles por huerto (con una carga mínima de 150 frutos) se tomaron cuatro frutos de cada uno. Posteriormente, de los mismos árboles se colectaron 12 frutos adicionales por huerto para determinar la materia seca de la pulpa, según la norma mexicana NMX-FF-016-SCFI (Secretaría de Economía, 2006). Antes del almacenamiento se registró el diámetro ecuatorial y la longitud de cada fruto. Los cuatro frutos de cada árbol se mantuvieron en una cámara ambiental (Lab-Line ${ }^{\circledR}$, modelo Biotronette Mark III, Kodamkulangara, India) a $22 \pm 1{ }^{\circ} \mathrm{C}$ y $85 \pm 10 \%$ de humedad relativa $(\mathrm{HR})$ hasta madurez de consumo.

Comportamiento postcosecha. Durante el almacenamiento en la cámara ambiental, cada 2 d y hasta madurez de consumo se registró la pérdida fisiológica de masa (PFM) con una balanza digital (Ohaus ${ }^{\circledR}$, CS2000, Parsippany, USA); los datos fueron expresados como porcentaje de masa perdida. El color en la piel fue medido cada $2 \mathrm{~d}$ por triplicado en la parte media del fruto con un colorímetro de reflectancia (HunterLab®, modelo Colorflex, Virginia, USA). Para determinar si los frutos alcanzaron la madurez de consumo, se obtuvieron los valores de luminosidad $(L *)$, cromaticidad (C*) y matiz $\left(h^{\circ}\right)$ (McGuire, 1992) al final del almacenamiento. El número de días para alcanzar la madurez de consumo se registró para todos los frutos y fue asignada a frutos que superaron el $75 \%$ de la piel virada a negro (Frutos 4 y 5 de la Figura 1).

Pruebas de aceptabilidad y preferencia. Se realizó una evaluación sensorial por un panel de 30 jueces adultos no entrenados de diversas edades (de 18 a 54 años). En 2012, en los muestreos de fruto del 12 y 26 de julio, así como del 9 y 23 de agosto, se realizaron las pruebas de aceptabilidad en frutos en madurez de consumo de los dos cultivares de aguacate. Se removió la piel de los frutos y la pulpa fue cortada en segmentos de $2 \times 2 \times 1 \mathrm{~cm}$ a los que se les asignó

Cuadro 1. Características de los huertos de aguacate empleados para el Estudio 1.

\begin{tabular}{lccccc}
\hline \multirow{2}{*}{ Características } & \multicolumn{5}{c}{ Huerto } \\
\cline { 2 - 5 } & Los Manantiales & Cerritos & Los Manantiales & Sesangari 5 & El Colorín 2 \\
\hline Cultivar & Hass & Méndez & Méndez & Méndez & Méndez \\
Altitud $(\mathrm{m})$ & 1633 & 1495 & 1632 & 1800 & 1624 \\
Suelo & Andosol & Cambisol & Andosol & Andosol & Andosol \\
Clima & $(\mathrm{A}) \mathrm{C}(\mathrm{w} 2)(\mathrm{w})^{\dagger}$ & $(\mathrm{A}) \mathrm{C}(\mathrm{w} 2)(\mathrm{w})^{\dagger}$ & $(\mathrm{A}) \mathrm{C}(\mathrm{w} 2)(\mathrm{w})^{\dagger}$ & $(\mathrm{A}) \mathrm{C}(\mathrm{m})(\mathrm{w})^{++}$ & $(\mathrm{A}) \mathrm{C}(\mathrm{m})(\mathrm{w})^{++}$ \\
Edad (años) & 15 & 5 & 6 & 5 & 10 \\
Distancias $(\mathrm{m})$ & $10 \times 10$ & $6 \times 4$ & $8 \times 4$ & $5 \times 3$ & $10 \times 10$ \\
Sistema de riego & Aspersión & Microaspersión & Aspersión & Microaspersión & Aspersión \\
\hline
\end{tabular}

†Semicálido subhúmedo con lluvias en verano; ${ }^{+\top}$ Semicálido húmedo con abundantes lluvias en verano. 


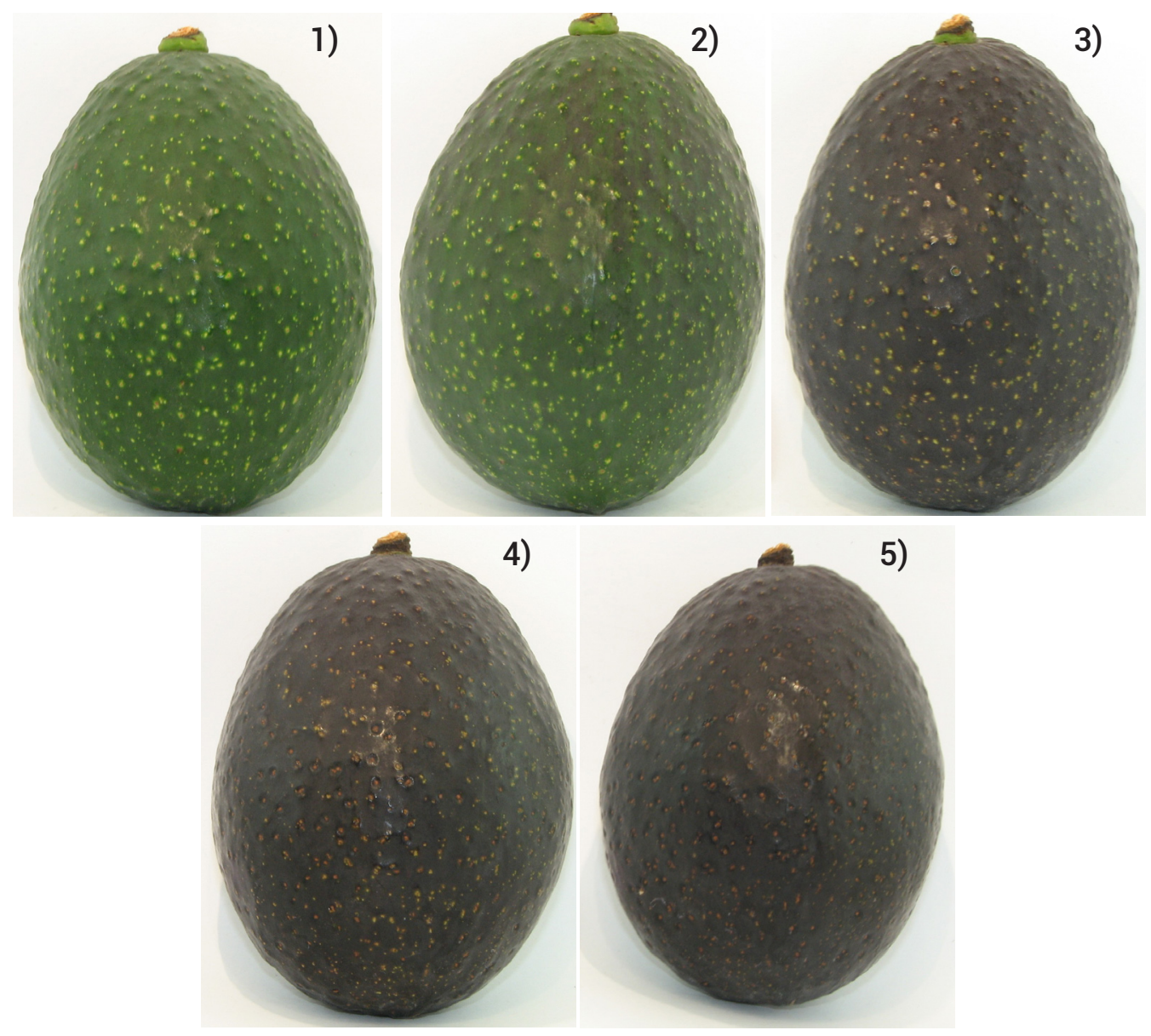

Figura 1. Escala de maduración de frutos de Méndez, según el cambio del color de la piel: 1) verde bosque; 2) fruto virado a negro $(25 \%) ; 3$ ) fruto virado a negro $(50 \%) ; 4)$ fruto virado a negro $(75 \%) ; 5)$ fruto $100 \%$ negro.

de manera aleatoria un código de tres dígitos. El panelista indicó su nivel de agrado de la pulpa en una escala hedónica de cinco puntos desde "me gusta mucho" a "me disgusta mucho". La descripción cuantitativa incluyó la evaluación del sabor, color, olor y textura. En el muestreo de fruto del 23 de agosto se realizó una prueba de preferencia de la pulpa entre los frutos procedentes de los cuatro huertos de Méndez. En este caso, el panelista asignó un valor entre 1 (el que más me agrada) y 4 (el que menos me agrada), según su preferencia (Obenland et al., 2012).

\section{Estudio 2. Respiración y producción de etileno}

La velocidad de respiración y producción de etileno fue cuantificada en 2013, en frutos originados por la floración de verano-otoño 2012 de dos huertos de Hass y dos de Méndez, ubicados en los municipios de Uruapan y Peribán de Ramos, Michoacán (Cuadro 2).

Muestreo y análisis de fruto. Se realizaron tres muestreos de fruto a intervalos de cuatro semanas y diferente estado de madurez (MS de la pulpa). El primero fue el 29 de julio (Hass $23.8 \%$; Méndez $24.9 \%$ ), el segundo el 26 de agosto (Hass $25.9 \%$; Méndez $26.4 \%$ ), y el tercero el 23 de septiembre (Hass y Méndez $28 \%$ ). En cada muestreo se colectaron 12 frutos por huerto y se dividieron en cuatro grupos de tres frutos cada uno para cada periodo de refrigeración: $0,7,14$ y $28 \mathrm{~d}$ a $5.5^{\circ} \mathrm{C} \pm 1^{\circ} \mathrm{C}$ y HR $90 \pm 5 \%$. Después de la refrigeración los frutos fueron trasladados a temperatura ambiente $\left(22 \pm 2{ }^{\circ} \mathrm{C}\right.$ y $\left.85 \pm 10 \% \mathrm{HR}\right)$ hasta madurez de consumo.

Comportamiento postcosecha. Durante la refrigeración y en temperatura ambiente se midió la velocidad de respiración $\left(\mathrm{mL} \mathrm{CO} \mathrm{kg}^{-1} \mathrm{~h}^{-1}\right)$ y la producción de etileno $\left(\mu \mathrm{Lgg}^{-1} \mathrm{~h}^{-1}\right)$, para lo cual se encerró un fruto por $2 \mathrm{~h}$ en un recipiente de vidrio hermético, al que se le adaptó una septa. Del espacio libre de cabeza se tomó $1 \mathrm{~mL}$ y se inyectó en un cromatógrafo de gases (Agilent ${ }^{\circledR} 6850$ Series II; Santa Clara CA, USA) acoplado a un espectrómetro de masas (Agilent ${ }^{\circledR}$ 5973) y una columna capilar de $30 \mathrm{~cm} \times 0.52 \mu \mathrm{m} \times 0.32 \mu \mathrm{m}(\mathrm{HP}-$

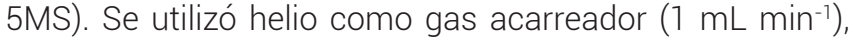


Cuadro 2. Características de los huertos de aguacate del Estudio 2.

\begin{tabular}{lcccc}
\hline \multirow{2}{*}{ Características } & \multicolumn{4}{c}{ Huerto } \\
\cline { 2 - 5 } & La Joya 16 & Llano Grande & Los Manantiales & Llano Grande \\
\hline Cultivar & Hass & Hass & Méndez & Méndez \\
Altitud $(\mathrm{m})$ & 2038 & 1449 & 1632 & 1449 \\
Suelo & Andosol & Cambisol & Andosol & Cambisol \\
Clima & $\mathrm{C}(\mathrm{m})(\mathrm{w})^{+}$ & $(\mathrm{A}) \mathrm{C}(\mathrm{w} 2)(\mathrm{w})^{++}$ & $(\mathrm{A}) \mathrm{C}(\mathrm{w} 2)(\mathrm{w})^{++}$ & $(\mathrm{A}) \mathrm{C}(\mathrm{w} 2)(\mathrm{w})^{++}$ \\
Edad (años) & 7 & 8 & 6 & 8 \\
Distanciamiento $(\mathrm{m})$ & $6 \times 4$ & $8 \times 8$ & $8 \times 4$ & $8 \times 8$ \\
Sistema de riego & Sin riego & Microaspersión & Aspersión & Microaspersión \\
\hline
\end{tabular}

${ }^{\dagger}$ Templado húmedo con abundantes Iluvias en verano; ${ }^{+\dagger}$ Semicálido húmedo con Iluvias en verano.

la temperatura del detector de $300{ }^{\circ} \mathrm{C}$ y la de inyección de $250^{\circ} \mathrm{C}$. El tiempo de retención fue de 10 min a una temperatura de $150^{\circ} \mathrm{C}$ y programado para aumentar la temperatura a una velocidad de $5^{\circ} \mathrm{C} \mathrm{min}^{-1}$ hasta una temperatura final de $278{ }^{\circ} \mathrm{C}$ por 5 min (López-Bucio et al., 2007). Las mediciones se realizaron cada $3 \mathrm{~d}$ para frutos en refrigeración y diariamente para los frutos a temperatura ambiente.

\section{Análisis estadístico}

En ambos estudios se utilizó un diseño experimental completamente al azar. En el primer estudio se tuvieron como factores de variación el cultivar y las fechas de muestreo, mientras que en el segundo los factores de variación fueron el cultivar y el estado de madurez del fruto en cada periodo de almacenamiento. Se realizaron análisis de varianza con el paquete estadístico SAS (SAS Instutite, 2010) V9.2 y la comparación de medias se realizó con la prueba de Waller-Duncan $(a=0.05)$. La evaluación de la aceptabilidad se analizó con la prueba de Mann-Whitney $(a=0.05)$, y la de preferencia con la prueba de KruskalWallis $(a=0.05)$.

\section{RESULTADOS Y DISCUSIÓN}

\section{Estudio 1. Madurez de cosecha}

Los dos cultivares alcanzaron la madurez fisiológica 10 meses después de antesis con una temperatura media anual entre 17.1 a $19.0^{\circ} \mathrm{C}$ en todos los huertos. En los muestreos del 31 de mayo, 14 de junio y 28 de junio los contenidos de MS fueron menores a lo establecido en la NMX-FF-016-SCFI (Secretaría de Economía, 2006), donde $21.5 \%$ de MS se considera como madurez fisiológica en aguacate Hass; además, la piel de los frutos de ambos cultivares no viró a negro ni alcanzaron la madurez de consumo durante su almacenamiento a temperatura ambiente y menos del $50 \%$ de los frutos que se colectaron en estos primeros muestreos maduró (Cuadro 3).

Cuadro 3. Materia seca (MS), frutos maduros y pérdida fisiológica de masa (PFM) de frutos de aguacate Hass y Méndez cosechados en 2012 y almacenados a temperatura ambiente $\left(22 \pm 1^{\circ} \mathrm{C}\right.$ y $\left.85 \pm 10 \% \mathrm{HR}\right)$ hasta madurez de consumo.

\begin{tabular}{|c|c|c|c|c|c|c|}
\hline \multirow{2}{*}{$\begin{array}{l}\text { Fecha de muestreo } \\
\text { (2012) }\end{array}$} & \multicolumn{2}{|c|}{ MS en la pulpa (\%) } & \multicolumn{2}{|c|}{ Frutos maduros $(\%)^{+}$} & \multicolumn{2}{|c|}{$\begin{array}{c}\text { PFM al final del } \\
\text { almacenamiento (\%) }\end{array}$} \\
\hline & Hass & Méndez & Hass & Méndez & Hass & Méndez \\
\hline 31 mayo & $18.3 \mathrm{~cd}^{++}$ & $20.0 \mathrm{~d}$ & $23 b$ & $17 \mathrm{~b}$ & $11.4 \mathrm{a}$ & $8.8 \mathrm{~b}$ \\
\hline 14 junio & $20.0 c$ & $21.0 \mathrm{~d}$ & $33 b$ & $10 \mathrm{~b}$ & $6.8 \mathrm{a}$ & $5.6 b$ \\
\hline 28 junio & $21.0 \mathrm{bc}$ & $21.1 \mathrm{~d}$ & $45 b$ & $25 b$ & $8.9 a$ & $6.9 \mathrm{~b}$ \\
\hline 12 julio & $21.1 \mathrm{bc}$ & $22.7 \mathrm{c}$ & $100 \mathrm{a}$ & $100 \mathrm{a}$ & $7.5 a$ & $5.4 b$ \\
\hline 26 julio & $23.6 \mathrm{ab}$ & $24.2 \mathrm{bc}$ & $100 \mathrm{a}$ & $100 \mathrm{a}$ & $8.4 \mathrm{a}$ & $6.2 \mathrm{~b}$ \\
\hline 09 agosto & $23.3 a b$ & $23.9 b$ & $100 \mathrm{a}$ & $100 \mathrm{a}$ & $6.9 a$ & $5.8 b$ \\
\hline 23 agosto & $26.3 \mathrm{a}$ & $27.3 a$ & $100 \mathrm{a}$ & $100 \mathrm{a}$ & $8.8 \mathrm{a}$ & $7.4 \mathrm{~b}$ \\
\hline
\end{tabular}

†Frutos que alcanzaron madurez de consumo entre el día 8 y 12 después de cosecha; ${ }^{+1}$ Medias con letras iguales en columnas no son estadísticamente diferentes (Waller-Duncan, $\mathrm{P} \leq 0.05$ ). 
En los primeros tres muestreos la luminosidad, cromaticidad y el matiz no presentaron cambios significativos en los dos cultivares, como normalmente sucede en la maduración de aguacate Hass cosechado en madurez adecuada (Dixon et al., 2004) (Cuadro 4; Figura 1). Cuando $L *, C * y h^{\circ}$ disminuyeron de manera notable, disminuyó la coloración verde y la piel viró a negro; entonces, la maduración ocurrió de manera regular y los frutos alcanzaron la madurez de consumo. Este cambio de color en la piel de Méndez, de verde a completamente negro fue similar en Hass, y coincide con lo reportado por Dixon et al. (2004).

En ambos cultivares de aguacate el cambio de color de la piel fue observado en todos los frutos a partir del muestreo del 12 de julio de 2012 (Cuadro 3). Además, todos los frutos alcanzaron la madurez de consumo entre 8 y $12 \mathrm{~d}$ después de la cosecha, lo cual fue similar a lo encontrado para Hass por Osuna-García et al. (2010). Sin embargo, esto no ocurrió en fechas de muestreo anteriores (Cuadro 3), lo que indica que los procesos fisiológicos para alcanzar la madurez de consumo ocurren de forma similar a Hass (Cox et al., 2004).
Hass presentó la mayor pérdida fisiológica de masa (PFM) en todas las fechas de muestreo para frutos almacenados a temperatura ambiente, con un promedio de 8.3 $\%$ en las siete fechas de muestreo, en comparación con Méndez que fue de $6.6 \%$ (Cuadro 3). La PFM afecta la apariencia del aguacate si supera el 10 \% (Herrera-González et al., 2013), pero ni Hass ni Méndez superaron este porcentaje.

Forma del fruto. Hass y Méndez no presentaron diferencias en la longitud del fruto, pero sí en el diámetro porque los frutos de Méndez fueron más redondeados que los de Hass (Cuadro 5; Figura 2). Otros cultivares tipo Hass también han presentado un diámetro mayor que esta variedad, como lo mostró Méndez en este estudio, aunque con pesos y MS superiores a los aquí reportados. En Nueva Zelanda, Lamb Hass con $24 \%$ MS y 318 g tuvo un diámetro de 7.4 cm y Gem con $28.9 \%$ MS y 233.2 g presentó un diámetro de $7.0 \mathrm{~cm}$ (Dixon et al., 2005; Dixon et al., 2008). Aunque los diámetros son superiores a los obtenidos, los autores mencionados no reportaron diferencias sobre la forma del fruto, como lo mostró Méndez en este estudio. La presencia de

Cuadro 4. Luminosidad, cromaticidad y matiz en la piel de frutos de aguacate Hass y Méndez cosechados de mayo a agosto de 2012 y almacenados a temperatura ambiente $\left(22 \pm 1^{\circ} \mathrm{C}\right.$ y $\left.85 \pm 10 \% \mathrm{HR}\right)$ hasta madurez de consumo.

\begin{tabular}{lcccccc}
\hline \multirow{2}{*}{$\begin{array}{l}\text { Fecha de } \\
\text { muestreo (2012) }\end{array}$} & Hass & Méndez & Hass & Méndez & Hass & Méndez \\
\cline { 2 - 7 } 31 mayo & ${ }^{*}$ A 27.9 a $\mathrm{a}^{++}$ & B 26.8 a & A 8.9 a & A 8.1 a & B 298.1 a & A 300.8 a \\
14 junio & A 27.0 a & A 26.5 a & A 7.8 a & A 7.6 ab & B 295.6 a & A 300.5 a \\
28 junio & B 25.0 b & A 26.3 a & B 5.2 b & A 7.2 b & A 173.0 b & B 282.7 a \\
12 julio & A 24.6 b & A 25.3 b & A 4.9 b & A 5.6 C & A 95.8 bc & A 125.2 C \\
26 julio & A 22.8 C & A 23.6 C & A 3.3 C & A 3.6 d & A 180.5 b & A 219.0 b \\
09 agosto & B 22.2 C & A 23.3 C & B 2.1 C & A 3.4 d & A 67.3 C & A 126.1 C \\
23 agosto & A 22.1 C & A22.4 d & A 1.9 C & A 2.19e & A 69.6 C & A 78.5 d \\
\hline
\end{tabular}

L*: luminosidad; $C *$ : $(a * 2+b * 2) 1 / 2]$ : Chroma; $h^{\circ}$ (arcotangente $\left.b * / a *\right)$ : ángulo hue [0 (valores positivos): rojo, $90^{\circ}$ : amarillo, $180^{\circ}$ (valores negativos): verde, 270: azul)] (McGuire, 1992); ${ }^{\dagger}$ Medias con letras mayúsculas iguales en las filas no son estadísticamente diferentes (Waller-Duncan, $\left.\mathrm{P} \leq 0.05\right)$;

${ }^{++}$Medias con letras minúsculas iguales en las columnas no son estadísticamente diferentes (Waller-Duncan, $\mathrm{P} \leq 0.05$ ).

Cuadro 5. Comparación de longitud, diámetro ecuatorial y relación diámetro/longitud de frutos de los aguacates Hass y Méndez.

\begin{tabular}{lcccc}
\hline Cultivar & $\begin{array}{c}\text { Peso } \\
(\mathrm{g})\end{array}$ & $\begin{array}{c}\text { Longitud } \\
(\mathrm{cm})\end{array}$ & $\begin{array}{c}\text { Diámetro } \\
\text { ecuatorial } \\
(\mathrm{cm})\end{array}$ & $\begin{array}{c}\text { Diámetro/ } \\
\text { Longitud }\end{array}$ \\
\hline Hass & $212.3 \mathrm{a}^{+}$ & $8.75 \mathrm{a}$ & $6.42 \mathrm{~b}$ & $0.73 \mathrm{~b}$ \\
Méndez & $225.6 \mathrm{a}$ & $8.86 \mathrm{a}$ & $6.72 \mathrm{a}$ & $0.77 \mathrm{a}$ \\
\hline
\end{tabular}

${ }^{\top}$ Medias con letras iguales en columnas no son estadísticamente diferentes (Waller-Duncan, $\mathrm{P} \leq 0.05$ ).
Cuadro 6. Grado de aceptación en una escala de 0 a 100 , de atributos sensoriales de la pulpa de frutos de aguacate Hass y Méndez en madurez de consumo. Promedio de las cuatro últimas cosechas de 2012 (12 de julio, 26 de julio, 9 de agosto y 23 de agosto).

\begin{tabular}{lcccc}
\hline \multirow{2}{*}{ Cultivar } & \multicolumn{4}{c}{ Grado de aceptabilidad (\%) } \\
\cline { 2 - 5 } & Color & Olor & Textura & Sabor \\
\hline Hass & $91.6 \mathrm{a}^{\dagger}$ & $82.8 \mathrm{a}$ & $84.6 \mathrm{a}$ & $88.4 \mathrm{a}$ \\
Méndez & $94.4 \mathrm{a}$ & $85.1 \mathrm{a}$ & $88.8 \mathrm{a}$ & $83.7 \mathrm{a}$ \\
\hline
\end{tabular}

${ }^{\top}$ Medias con letras iguales en columnas no son estadísticamente diferentes (Mann-Whitney, $\mathrm{P} \leq 0.05$ ). 

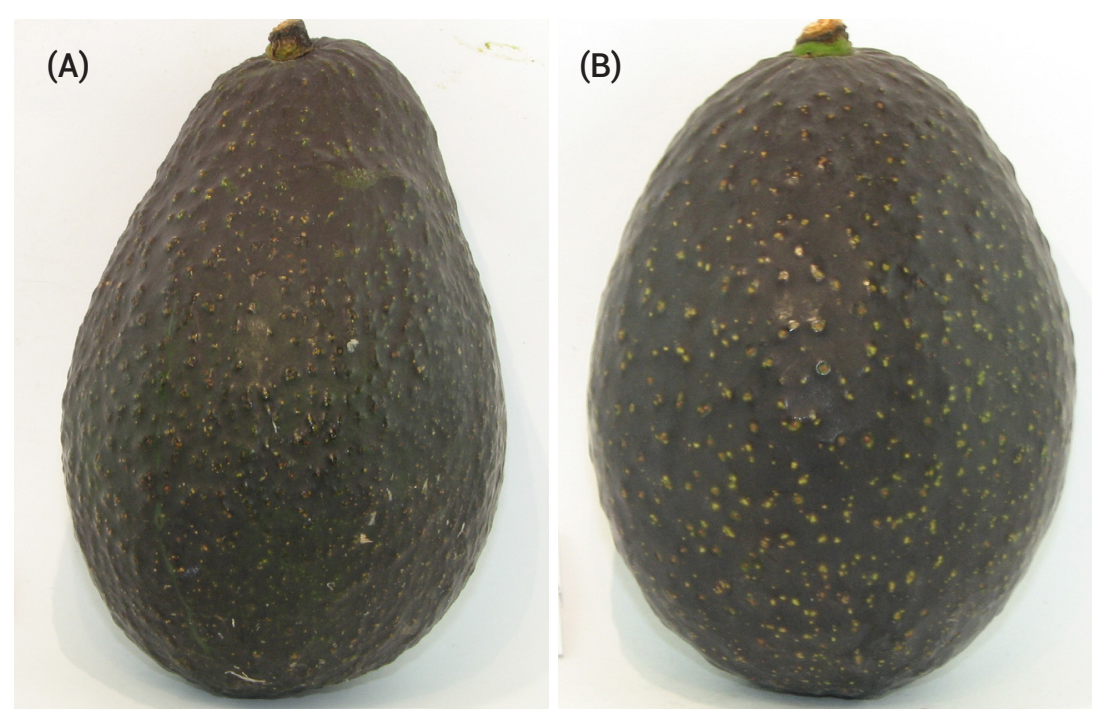

Figura 2. Forma del fruto de los aguacates Hass (A) y Méndez (B).

Cuadro 7. Nivel de agrado de la pulpa, en una escala hedónica de cinco puntos con un rango de "me gusta mucho" a "me disgusta mucho", en frutos de aguacate Méndez procedentes de cuatro huertos (cosecha: 23 de agosto 2012).

\begin{tabular}{llll}
\hline Escala & Descripción & Huerto & \\
\hline 1 & Me agrada mucho & Sesangari 5 & $\mathrm{a}^{\dagger}$ \\
2 & Me agrada & El Colorín & $\mathrm{b}$ \\
& & Los Manantiales & $\mathrm{b}$ \\
3 & Me desagrada & Cerritos & $\mathrm{c}$ \\
4 & Me desagrada mucho & --- & \\
\hline
\end{tabular}

${ }^{\dagger}$ Huertos con letras iguales no son estadísticamente diferentes (Kruskal-Wallis, $\mathrm{P} \leq 0.05$ ).

frutos más redondeados en Méndez que en Hass pudiera ser debida a la interacción cultivar-portainjerto o al manejo cultural que puede afectar algunas características del fruto como tamaño, forma, tolerancia a enfermedades y vida postcosecha (Willingham et al., 2004).

Prueba de aceptabilidad. Los frutos de Hass y Méndez cosechados en madurez fisiológica (después del 12 de julio de 2012) tuvieron aceptabilidad similar en todos los aspectos evaluados (Cuadro 6). Cuando los frutos cosechados el 26 de julio de 2012 alcanzaron la madurez de consumo, Méndez (24.2 \% MS) presentó mayor aceptabilidad entre los panelistas que Hass (23.6 \% MS) en color, olor y textura de la pulpa, pero en el sabor de la pulpa fueron similares.

Pruebas de preferencia. En el último muestreo realizado para el cv. Méndez (23 de agosto de 2012), los frutos de los cuatro huertos presentaron diferencias en la preferen- cia de los panelistas para las características sensoriales. El fruto del huerto Sesangari 5 (1800 msnm) presentó mejores características sensoriales, porque $52 \%$ de los panelistas lo ubicaron en primer lugar (Cuadro 7). Los frutos del huerto Cerritos (1632 msnm) fueron los de menor agrado sensorial según $44 \%$ de los panelistas. En el caso de los huertos Los Manantiales y El Colorín 2, sus frutos tuvieron preferencia intermedia (Cuadro 7).

El sabor característico de la pulpa de Hass no sólo está asociado con el contenido de aceite, sino también con la velocidad de respiración y la producción de etileno (Obenland et al., 2012), así como con los compuestos volátiles (Bayarri et al., 2006). Las diferencias en las pruebas de aceptabilidad mostradas por Hass y Méndez pudieran deberse a la gran cantidad de atributos que potencian el sabor del fruto durante la precosecha, como la ubicación geográfica y las prácticas culturales (Obenland et al., 2012). La ausencia de diferencias sensoriales entre la pulpa de Hass y Méndez permite suponer que los procesos de desarrollo y maduración son similares para ambos cultivares. Esto confiere a Méndez ventajas para su comercialización y transporte.

\section{Estudio 2. Respiración y producción de etileno}

La producción de etileno y la respiración fueron similares entre Hass y Méndez para cada fecha de muestreo y periodo de refrigeración (Figura 3). En frutos de la primera cosecha de Hass (23.8 \% MS) y Méndez (24.9\% MS) almacenados a temperatura ambiente, el climaterio se alcanzó 7 a 8 d después de la cosecha (Figura 3). Frutos cosechados con mayor MS (tercer muestreo) presentaron mayor producción de etileno y mayor velocidad de respiración. (Figura 3). 

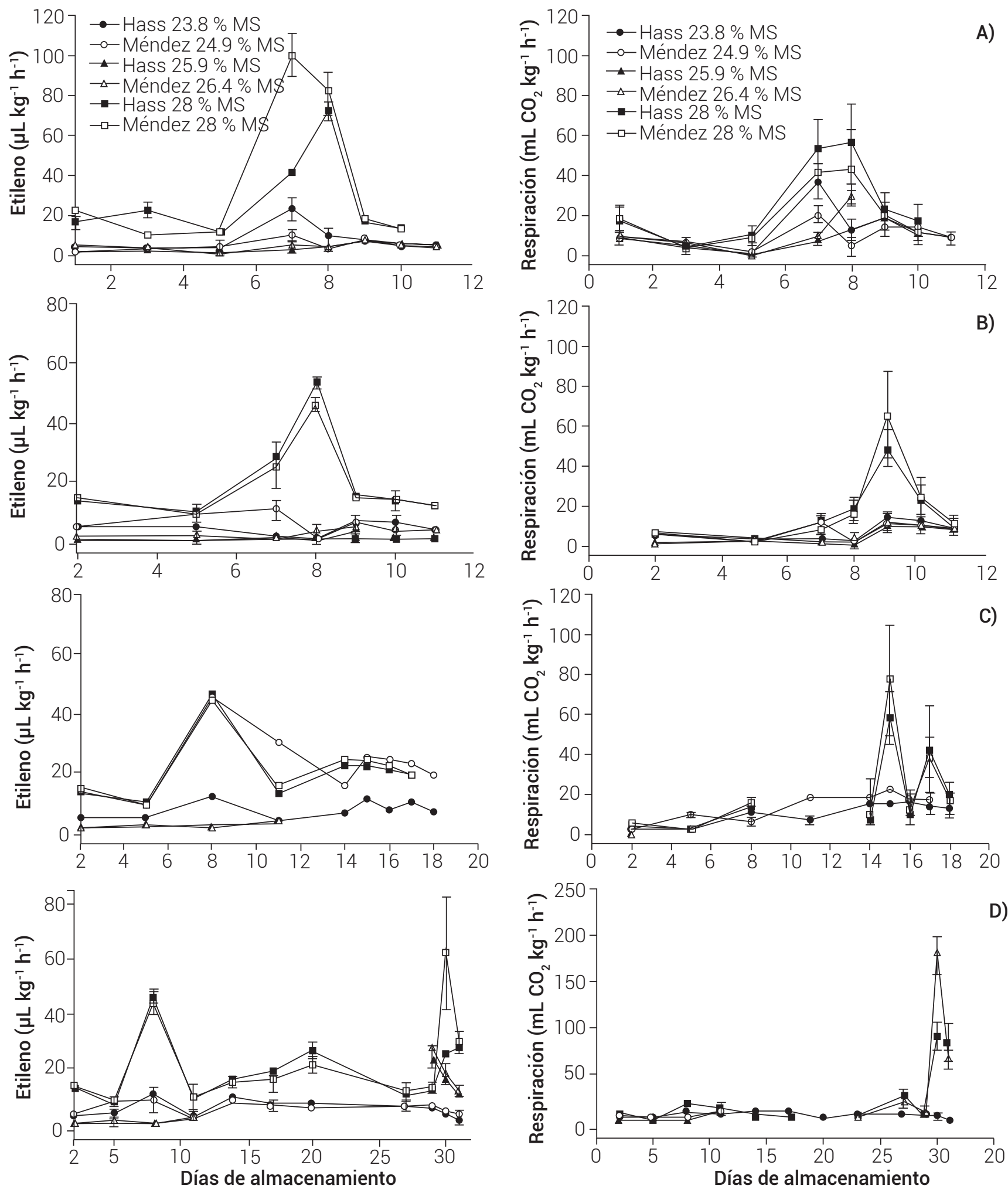

Figura 3. Producción de etileno y velocidad de respiración de frutos de Hass y Méndez cosechados en diferente estado de madurez (\% MS: materia seca) y almacenados por 0 (A), 7 (B), 14 (C) y 28 (D) d en refrigeración y posterior traslado a temperatura ambiente. Los datos representan la media de tres valores y la desviación estándar. 
Después de $7 \mathrm{~d}$ de refrigeración y posterior traslado a temperatura ambiente, los frutos de ambos cultivares y de todos los muestreos realizados presentaron un pico de producción de etileno. La mayor velocidad de respiración y producción de etileno ocurrió de 1 a 2 d después del traslado a temperatura ambiente (Figura 3).

La velocidad de respiración y producción de etileno fue similar para los aguacates Hass y Méndez refrigerados durante $14 \mathrm{~d}$. La producción de etileno en frutos cosechados en el último muestreo (28\% MS) alcanzó el climaterio un día después del traslado a temperatura ambiente; descendió al día siguiente y tuvo otro incremento menor un día después (Figura 3).

La producción de etileno y la velocidad de respiración en frutos refrigerados durante $28 \mathrm{~d}$ fueron similares para los dos cultivares. En estas condiciones, ambos cultivares presentaron un ligero incremento en la producción de etileno. Los dos cultivares tuvieron su mayor producción de etileno después del traslado a temperatura ambiente. La velocidad de respiración en frutos del primer muestreo (Méndez 24.9 \% MS y Hass 23.8 \% MS) fue la más baja de todos los muestreos y de todos los periodos de almacenamiento, tanto en refrigeración como en temperatura ambiente (Figura 3).

Méndez presentó una velocidad de respiración y producción de etileno similar a Hass. Para el primer muestreo de fruto (< $25 \% \mathrm{MS}$ ) en ambos cultivares se obtuvo baja producción de etileno y baja velocidad de respiración; sin embargo, lo contrario ocurrió en las cosechas siguientes (> $26.4 \% \mathrm{MS}$ ). Aunque la mayor producción de etileno y velocidad de respiración ocurrió posterior al traslado a temperatura ambiente, ambos cultivares presentaron otros picos de producción entre los días ocho y 10 de refrigeración. La producción de etileno y velocidad de respiración registrados fueron similares a los mencionados por Dixon et al. (2003), Hershkovitz et al. (2009a y b) y Osuna-García et al. (2010).

\section{CONCLUSIONES}

La madurez fisiológica difirió entre ambos cultivares, Méndez (22.7 \% MS) y Hass (21.7 \% MS). Los parámetros de calidad evaluados a la cosecha, en postcosecha y en madurez de consumo fueron similares cuando el fruto de ambos cultivares fue cosechado en madurez fisiológica. La velocidad de respiración y la producción de etileno en refrigeración de frutos de Hass y Méndez por 7, 14 y 28 d a $5.5^{\circ} \mathrm{C} \pm 1{ }^{\circ} \mathrm{C}$ y HR $90 \pm 5 \%$ y a temperatura ambiente $\left(22{ }^{\circ} \mathrm{C} \pm 2{ }^{\circ} \mathrm{C}\right.$ y HR $\left.85 \pm 10 \%\right)$ fueron similares cuando se cosecharon en madurez fisiológica.

\section{BIBLIOGRAFÍA}

Bayarri S., A. J. Taylor and J. Hort (2006) The role of fat in flavor perception: effect of partition and viscosity in model emulsions. Journal of Agricultural and Food Chemistry 54:8862-8868.

Cox K. A., T. K. McGhie, A. White and A. B. Woolf (2004) Skin colour and pigment change during ripening of Hass avocado fruit. Postharvest Biology and Technology 31:287-294.

Dixon J., C. Cotterell, B. Hofstee and T. A. Elmsly (2008) University of California avocado cultivars 'Lamb Hass' and 'Gem' maturity and fruit quality results from New Zealand evaluation trials. New Zealand Avocado Growers' Association Annual Research Report 8:15-26.

Dixon J., T. A. Elmsly, D. B. Smith and H. A. Pak (2005) Increasing pick to pack times increases ripe rots in Hass avocados. New Zealand Avocado Growers' Association Annual Research Report 5:4350.

Dixon J., H. A. Pak, D. B. Smith, T. A Elmsly and J. G. M. Cutting (2003) New Zealand avocado fruit quality: the impact of storage temperature and maturity. In: Proceedings $\vee$ World Avocado Congress. 19 -24 Oct. Granada. Málaga, Spain. pp:647-652.

Dixon J., D. B. Smith and T. A. Elmsly (2004) Quality of Hass avocado (Persea americana Mill.) fruit after high humidity storage in polyethylene bags. New Zealand Avocado Growers' Association Annual Research Report 4:54-60.

Ernst A. (2007) Maluma Hass ${ }^{\circledR}$ : a new released cultivar in comparison with Hass. In: Proceedings VI World Avocado Congress. 12-16 Nov. Viña Del Mar, Chile. pp:1-8.

Herrera-González J. A., S. Salazar-García, P. Gutiérrez-Martínez e I. J. L. González-Durán (2013) El comportamiento poscosecha de frutos de aguacate Hass es influenciado por el portainjerto. Revista Mexicana de Ciencias Agrícolas 4:19-32.

Hershkovitz V., H. Friedman, E. E. Goldschmidt, O. Feygenberg and E. Pasis (2009a) Induction of ethylene in avocado fruit in response to chilling stress on tree. Journal of Plant Physiology 166:18551862.

Hershkovitz V., H. Friedman, E. E. Goldschmidt and E. Pasis (2009b) The role of the embryo and ethylene in avocado fruit mesocarp discoloration. Journal of Experimental Botany 60:791-799.

Huysamer M. and L. Maré (2003) The effect of relative humidity and ethylene scrubbing on 'Fuerte' and Hass avocado fruit quality. South African Avocado Growers' Association Yearbook 26:96-105.

Illsley-Granich C., R. Brokaw and S. Ochoa-Ascencio (2011) Hass Carmen ${ }^{\circledR}$, a precocious flowering avocado tree. In: Proceedings VII World Avocado Congress. 5-9 Sep. Cairns, Australia. pp:1-6.

López-Bucio J., J. C. Campos-Cuevas , E. Hernández-Calderón, C. VelásquezBecerra, R. Farías-Rodríguez, L. I. Macías-Rodríguez, and E. ValenciaCantero (2007) Bacillus megaterium rhizobacteria promote growth and alter root-system architecture through an auxinand ethylene-independent signaling mechanism in Arabidopsis thaliana. Molecular Plant-Microbe Interactions 20:207-217.

McGuire R. G. (1992) Reporting of objective color measurements. HortScience 27:1254-1255

Obenland D., S. Collin, J. Sievert, F. Negm and M. L. Arpaia (2012) Influence of maturity and ripening on aroma volatiles and flavor in Hass avocado. Postharvest Biology and Technology 71:41-50

Osuna-García J. A., G. Doyon, S. Salazar-García, R. Goenaga and I. J. L. González-Durán (2010) Effect of harvest date and ripening degree on quality and shelf life of Hass avocado in Mexico. Fruits 65:367-375.

Salazar-García S., L. Zamora-Cuevas and R. J. Vega-López (2005) Update on the avocado industry of Michoacán, México. California Avocado Society Yearbook 87:31-44.

SAS Institute (2010) The SAS system for Windows. Release 9.2. SAS Institute. Cary, North Carolina, U.S.A.

Secretaría de Economía (2006) Productos alimenticios no industrializados para uso humano-fruta fresca-Aguacate (Persea americana Mill). Especificaciones. Diario Oficial de la Federación. Secretaría de Economía. http://2006-2012.sagarpa.gob.mx/ agronegocios/Lists/Instrumentos Tcnicos Normalizacin y 
Marcas Colecti/Attachments/67/NMX_AGUACATE.pdf (Agosto 2016).

Yearsley C., N. Lallu, J. Burdon, D. Billing, M. Punter and S. Osman (2002) Effects of prepacking holding temperatures on shelf life quality of Hass avocados. New Zealand Avocado Growers' Association
Annual Research Report 2:108-117.

Willingham S. L., L. M. Coates, A. W. Cooke and J. R. Dean (2004) Tree vigour influences disease susceptibility of Hass avocado fruits. Australasian Plant Pathology 33:17-21. 